\title{
Extensively Drug-Resistant Tuberculosis: A Case Report and Literature Review
}

\author{
João Alves de Araújo-Filho ${ }^{1,2}$, Arioldo Carvalho Vasconcelos-Jr ${ }^{1}$, Eduardo Martins de Sousa ${ }^{1}$, Colombina da Silveira ${ }^{2}$, Elisangela \\ Ribeiro $^{3}$, André Kipnis ${ }^{1}$ and Ana Paula Junqueira-Kipnis ${ }^{1}$ \\ ${ }^{1}$ Institute of Tropical Pathology and Public Health of Federal University of Goiás; ${ }^{2}$ Tropical Diseases Hospital Dr. Anuar Auad; ${ }^{3}$ Araújo Jorge \\ Hospital, Associação de Combate ao Câncer; Goiás, GO, Brazil
}

\begin{abstract}
Extensively drug-resistant tuberculosis (XDR-TB) is an emerging health problem that threatens tuberculosis (TB) control worldwide, since suitable treatment for this disease has not yet been found. We report a case of secondary pulmonary XDR-TB in a 54-year-old, HIV-negative male from Goiânia, Brazil. The patient had long-standing pulmonary tuberculosis (nine years) with extensive bilateral lung damage and had been treated with multiple antituberculosis drugs (self-administered) before XDR-TB diagnosis. The strain of Mycobacterium tuberculosis was resistant to R- rifampicin, H-isoniazid, E-ethambutol, Eto-ethionamide, Ofx-ofloxacin, and Am-amikacin. This patient died with multiple organ failure due to sepsis secondary to bacterial pneumonia.

Key-Words: Extensively drug-resistant tuberculosis, XDR-TB, tuberculosis, Mycobacterium tuberculosis.
\end{abstract}

Tuberculosis (TB) remains a major world health problem. Around two billion people are infected with Mycobacterium tuberculosis, the causal agent of this disease [1-3]. This accounts for a third of the total world population; it is estimated that nine million people become infected each year. Tuberculosis also contributes to two million deaths per year. The emergence of multi-drug resistant tuberculosis (MDRTB), particularly in the 1990s, has become an important health problem and threatens TB control worldwide [1-4]. The Center for Diseases Control and Prevention (CDC) and World Health Organization (WHO) concluded that $20 \%$ of the 17,690 strains analyzed worldwide from 2000 to 2004 were multi-drug resistant (MDR), resistant to at least rifampicin (R) and isoniazid $(\mathrm{H})$ ) and $2 \%$ were extensively drug-resistant (XDR$\mathrm{TB}$ ), that is, resistant to $\mathrm{R}$ and $\mathrm{H}$ and resistant to a fluoroquinolone and one or more of the following injectable drugs: amikacin (Am), capreomycin (Cm), or kanamycin (Km) [5,6]. The Stop TB partnership estimates that around 458,000 MDR-TB cases exist. [5]. As of November 2007, 41 countries have reported confirmed XDR-TB cases to WHO [7].

The global threat of XDR tuberculosis has great importance for public health and TB control. The existence of XDR-TB cases is a reflection of weaknesses in tuberculosis management. Correct diagnosis and treatment of TB cases are both fundamental in preventing the emergence of drug resistance.

Despite the relevance of XDR-TB in the worldwide TB scenario, clinical reports of XDR-TB cases are uncommon. We present a case of secondary XDR-TB in a male with pulmonary TB in a central state of Brazil. Informed consent was obtained from the patient's relatives.

Received on 22 May 2008; revised 18 October 2008.

Address for correspondence: Dr. João Alves de Araújo Filho. Instituto de Patologia Tropical e Saúde Pública da Universidade Federal de Goiás. Rua Delenda Rezende de Melo, S/No., Setor Universitário, Goiânia, Go, Brazil. Zip code: 74605-050. Phone: 5562 3209-6126. Fax: 5562 3521-1839. E-mail: araujofilho63@click21.com.br.

The Brazilian Journal of Infectious Diseases 2008;12(5):447-452. (C) 2008 by The Brazilian Journal of Infectious Diseases and Contexto Publishing. All rights reserved.

\section{Case Report}

A 54-year-old, HIV-negative male with a tuberculin skin test (TST) result of $32 \mathrm{~mm}$ from Goiânia, State of Goiás, Brazil was diagnosed with pulmonary TB in January 1998. He underwent a standard Brazilian anti-TB regimen (all treatments were self-administered), consisting of two months of treatment with R, H, and pyrazinamide (Z), followed by four months of $\mathrm{R}$ and H. In March 1999, he was diagnosed with pulmonary TB recurrence and began treatment with $\mathrm{R}, \mathrm{H}, \mathrm{Z}$, and ethambutol (E), the Brazilian standard regimen for TB recurrence. At the same time, an isolated culture sensitivity test was performed, showing resistance to $\mathrm{R}$ and $\mathrm{H}$. In May 1999, treatment was initiated with streptomycin (S), E, ethionamide (Eto), and Z (standard regimen for patients failing to respond to the former regimens). The patient did not tolerate Eto, and was switched to ofloxacin (Ofx). After three months of therapy, S and Z were stopped. As sputum smear and culture remained positive in July 2000, amikacin (Am), Ofx, E, terizidone (Trd), and clofazimine (Cfz) were started, as part of a trial for MDR-TB within the Brazilian TB program. Due to a cutaneous hypersensivity reaction to Trd, treatment was discontinued and subsequently restarted without Trd. In May 2001, Am treatment was stopped. During this whole period, the patient reported regular ingestion of the drugs, although they were self-administered. In January 2004, his attending physician decided to stop the MDR regimen and started $\mathrm{R}, \mathrm{H}, \mathrm{Z}$, and $\mathrm{E}$ once more due to failure of the MDR regimen. In November 2006, a new attempt at treatment was made with Am, Ofx, Clr, $\mathrm{Z}$, and Trd. Figure 1 summarizes the use of antituberculosis drugs. The patient's major signs and symptoms during followup were cough, fever, hemoptysis, progressive dyspnea, and weight loss (14 Kg below baseline).

In April 2002, a lower right pulmonary lobectomy was performed, in an attempt to improve the patient's prognosis. The surgery was complicated by tuberculous empyema and bronchopleural fistula.

During the follow-up period, the patient was hospitalized on four separate occasions, usually due to respiratory complications. His last hospitalization extended for 23 days, due to respiratory failure secondary to bacterial pneumonia, 
evolving to sepsis, multiple organ failure, and death. During the last hospitalization, the patient's hemogram showed erythrocytes - 5.46 tera/L, hematocrit - $40.7 \mathrm{~mL} / \mathrm{dL}$, hemoglobin - $12.2 \mathrm{~g} / \mathrm{dL}, 13,100$ leucocytes/ $\mathrm{mm}^{3}$ (97\% neutrophils) and 219,000 platelets $/ \mathrm{mm}^{3}$. The albumin level was $2.3 \mathrm{~g} / \mathrm{dL}$. Alanine aminotransferase (ALT) and aspartate aminotranferase (AST) tests, as well as urea and creatinine levels, were normal at admission, although the patient eventually developed renal failure.

Figure 2 shows a plain chest X-ray film from October 2006.

Peripheral blood mononuclear cells were isolated from the patient's blood for analysis of tuberculosis-specific cellular immune responses. CD4+ T cell responses against two $M$. tuberculosis proteins, ESAT6 and GLcB, were detected, but at low levels (Figure 3).

Table 1 shows the resistance profile during the patient's follow-up. The sensitivity tests were performed using the Canetti method, the indirect method, and with an MB/BacT automated test. The last susceptibility test report was available post mortem.

\section{Discussion}

XDR-TB is a worrisome threat for TB control worldwide. The dangerous potential of XDR-TB is apparent from a recent epidemic in a rural area in KwaZulu Natal, South Africa, where, from January 2005 to March 2006, 53 cases of XDR-TB were found among 221 cases of MDR-TB. All of the XDR-TB patients tested for HIV $(n=44)$ were positive, and 52 of the 53 XDR-TB patients died, with a median survival of 16 days from time of diagnosis for the 42 patients with confirmed time of death [8]. Kim at al (2007) [9] found in a South Korean cohort of patients with MDR-TB $(n=211)$ that the presence of extensive drug resistance, comorbidity, and hypoalbuminemia were independent poor prognosis factors in non-HIVinfected patients with MDR-TB. In a pilot program to treat MDR-TB ( $\mathrm{n}=108)$ in Uzbekistan, poor clinical condition and baseline second-line resistance contributed to treatment failure or death; among six patients failing treatment and who remained alive, five were found likely to be infected with XDR-TB strains [10].

$\mathrm{XDR}-\mathrm{TB}$ is a man-made problem. Inadequate drug treatment will select for drug-resistant strains that subsequently proliferate and become MDR strains (secondary MDR-TB), which eventually could be transmitted to contact cases, who acquire primary MDRTB. MDR-TB precedes XDR-TB. XDR strains emerge in a similar way; that is, if a patient fails to respond to a firstline drug regimen, then using an inadequate second line drug regimen will select for extensively drug-resistant strains (secondary XDR-TB), which in turn could be transmitted to contacts, causing primary XDR-TB cases [4,11-13]. Inadequate drug treatment can be associated with inadequate regimes, to inadequate supply/quality drugs, and/or inadequate drug intake [4]. The administration of directly observed therapy (DOT) for Mycobacterium tuberculosis leads to a significant reduction in the frequency of primary drug resistance and acquired drug resistance $[14,15]$.

The Brazilian Health Ministry adopted standardized regimens of antituberculosis treatment containing $R$ in the 1970's, free of charge for all TB patients, has implemented an efficient drug quality control program and has also recommended that state health departments adopt DOT. DOT has not been universally implemented by Brazilian states and towns, despite the federal recommendation. To date, Goiás state and the Goiânia municipality have not fully implemented DOT programs. Certainly, the self-administered treatment used by our patient during his illness played a major role in the emergence of this extensive pattern of resistance, since regular intake of the drugs could not be confirmed.

An emerging concern is that inadequate use of secondline drugs in MDR programs could lead to the emergence of XDR cases [10]. It is fundamental that MDR treatment be administered by specialized health care providers, due to its complexity, and directly-observed therapy should be guaranteed

An important issue concerning MDR-TB control and prevention of the emergence of XDR-TB is adequate training for health care providers in order to deal with the complex problem of the management of TB resistance. The Brazilian TB Control Program, through the National Reference Center's Professor Hélio Fraga, began providing update courses in MDR-TB in March 2005, for physicians, nurses, pharmacists, microbiologists, social workers, and TB program managers from all national regions. Additionally, the program adopted a standard regimen of MDR-TB treatment, which includes Am (or S), Ofx, Trd, E, and Z for at least 18 months [16].

The actual Brazilian definition of MDR-TB implies the detection of resistance to a third drug besides $\mathrm{R}$ and $\mathrm{H}$ or failure of the standard Brazilian failure regimen (S, E, Eto and Z), which occurred with this patient [16]. This definition probably underestimates the prevalence of MDR-TB; consequently, we believe the definition should be revised.

The hospital environment has proven to be a favorable place to disseminate XDR M. tuberculosis strains. Adequate nosocomial infection control strategies should include air extractors and windows, HEPA filters, UV lights, negative pressure rooms, and personal protection for health staff, such as the use of $\mathrm{N}-95$ masks $[8,17,18]$.

There are no suitable treatment regimens for XDR-TB, as this organism is resistant to first-line and second-line antituberculosis drugs, and WHO guidelines recommend the use of at least four drugs for patients with MDR-TB [4]. Thus, the management of XDR-TB cases remains controversial. The role of third-line drugs, such as linezolid, amoxicilin/clavulanate, and macrolides, in association with first and second-line drugs to which the TB organisms are still susceptible, was not evaluated. In spite of the urgent need, new classes of antituberculosis drugs are unlikely to become available for clinical use in the near future [18-21]. 
Table 1. Susceptibility pattern to antituberculosis drugs during follow-up of the tuberculosis patient.

\begin{tabular}{lcccc}
\hline Date & April 1999 & August 2002 & November 2006 & January 2007 \\
\hline Drug resistance & R, H & R, H, E & R, H, E, Et & R, H, E, Et, Ofx, Am \\
\hline
\end{tabular}

R- rifampicin; H- isoniazid; E- ethambutol; Eto- ethionamide; Ofx- ofloxacin; Am- amikacin.

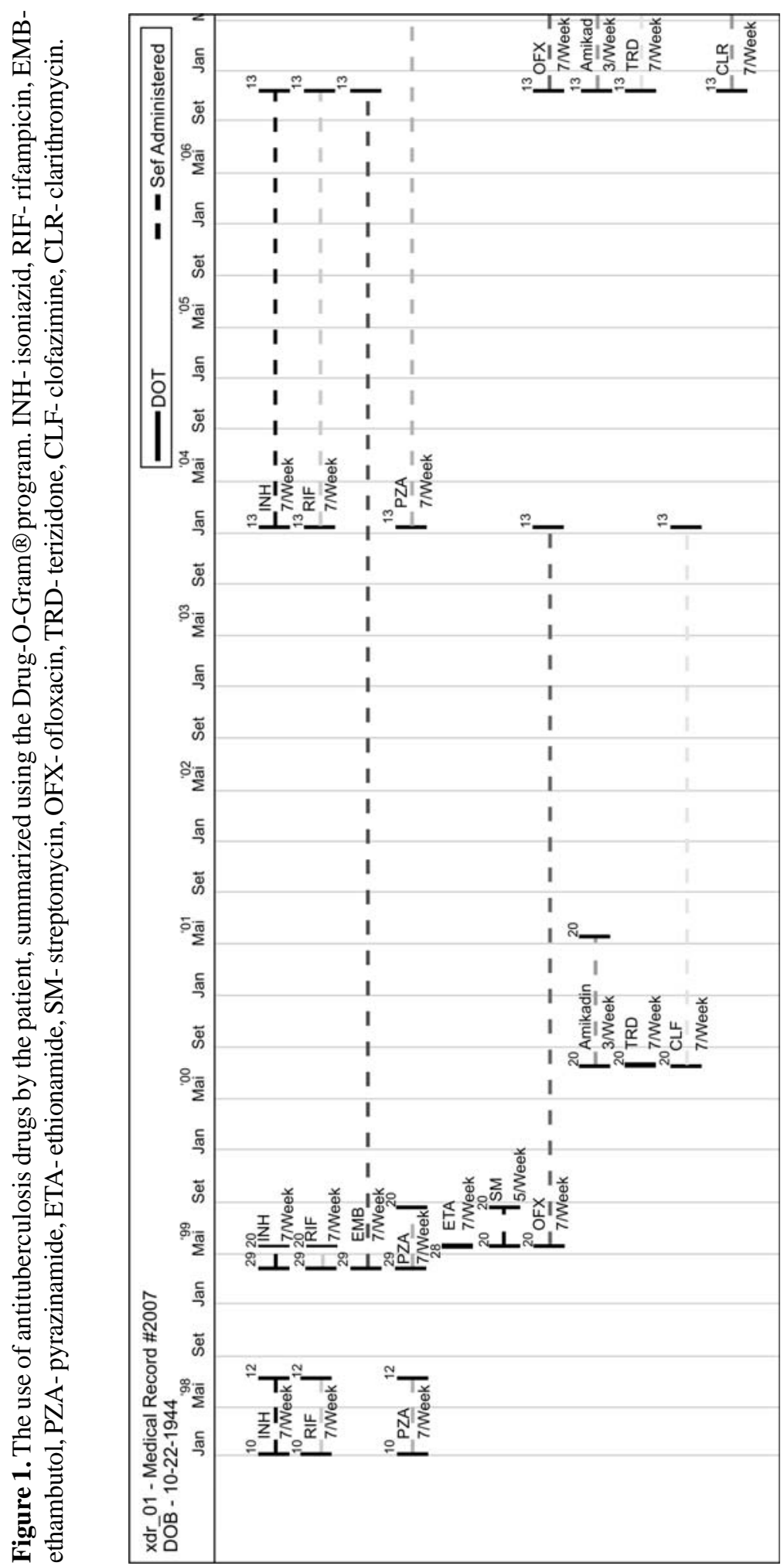

www.bjid.com.br 
Figure 2. Chest radiography showing an enormous cavern in the upper left lobe, with thickening of the right pleura and rough opacities and volumetric reduction of the right lung.

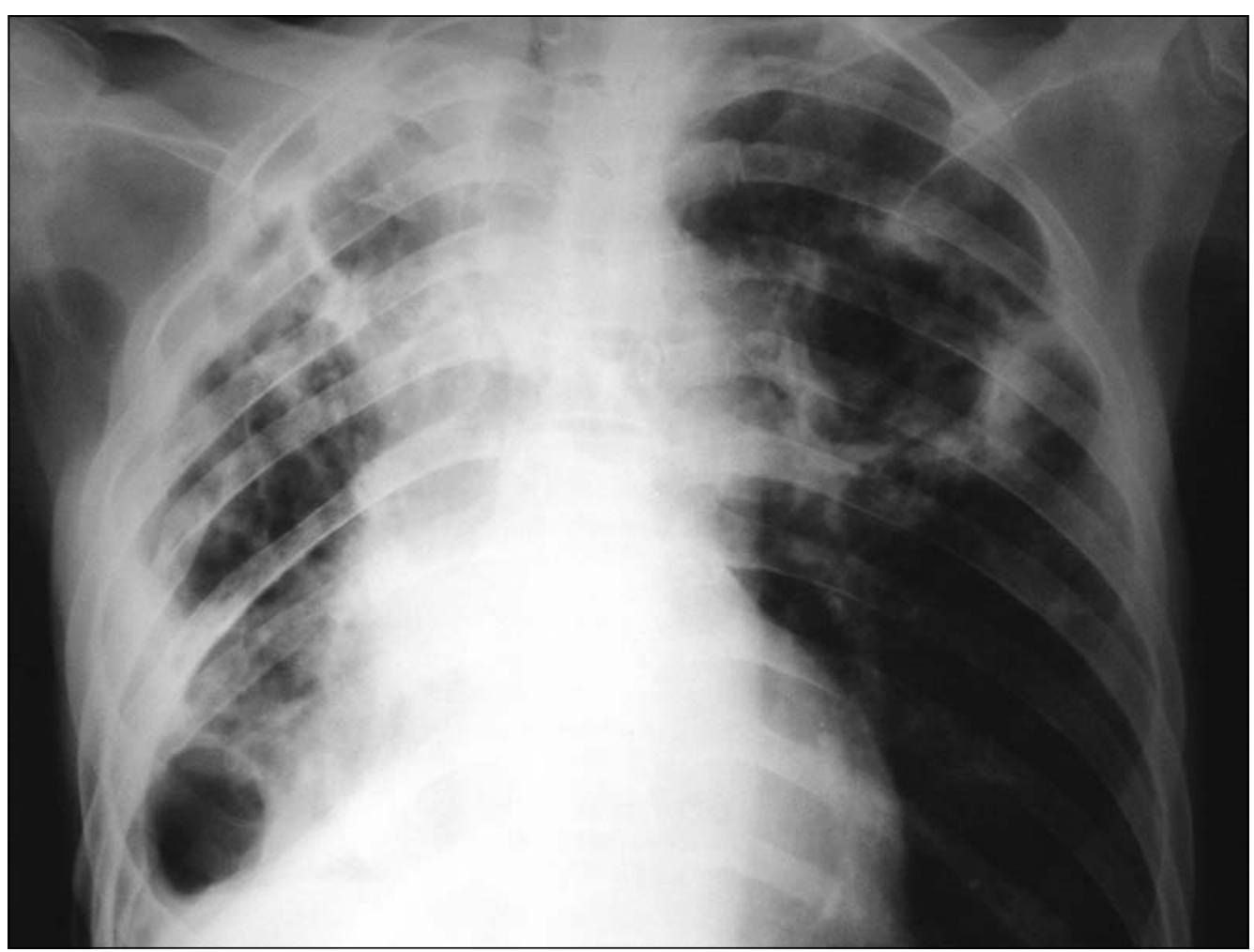

Figure 3. Flow cytometry analysis of Mycobacterium tuberculosis-specific CD4 ${ }^{+} \mathrm{T}$ cell responses (intracellular cytokine production by $\mathrm{CD}^{+}$T cells) in the peripheral blood of the patient with XDR-TB. An intracellular cytokine-staining assay was performed using freshly-isolated peripheral blood mononuclear cells stimulated for 48 hours with ESAT-6 and GLcB recombinant proteins of $M$. tuberculosis. The percentages in the upper right quadrants indicate the percentage of CD4 ${ }^{+}$cytokine-positive cells. For the purpose of comparison, results are shown from a 56-year-old, non-resistant TB patient with a TST of 12 mm. IFN$\gamma$ : interferon- $\gamma$; IL-10: interleukin-10.

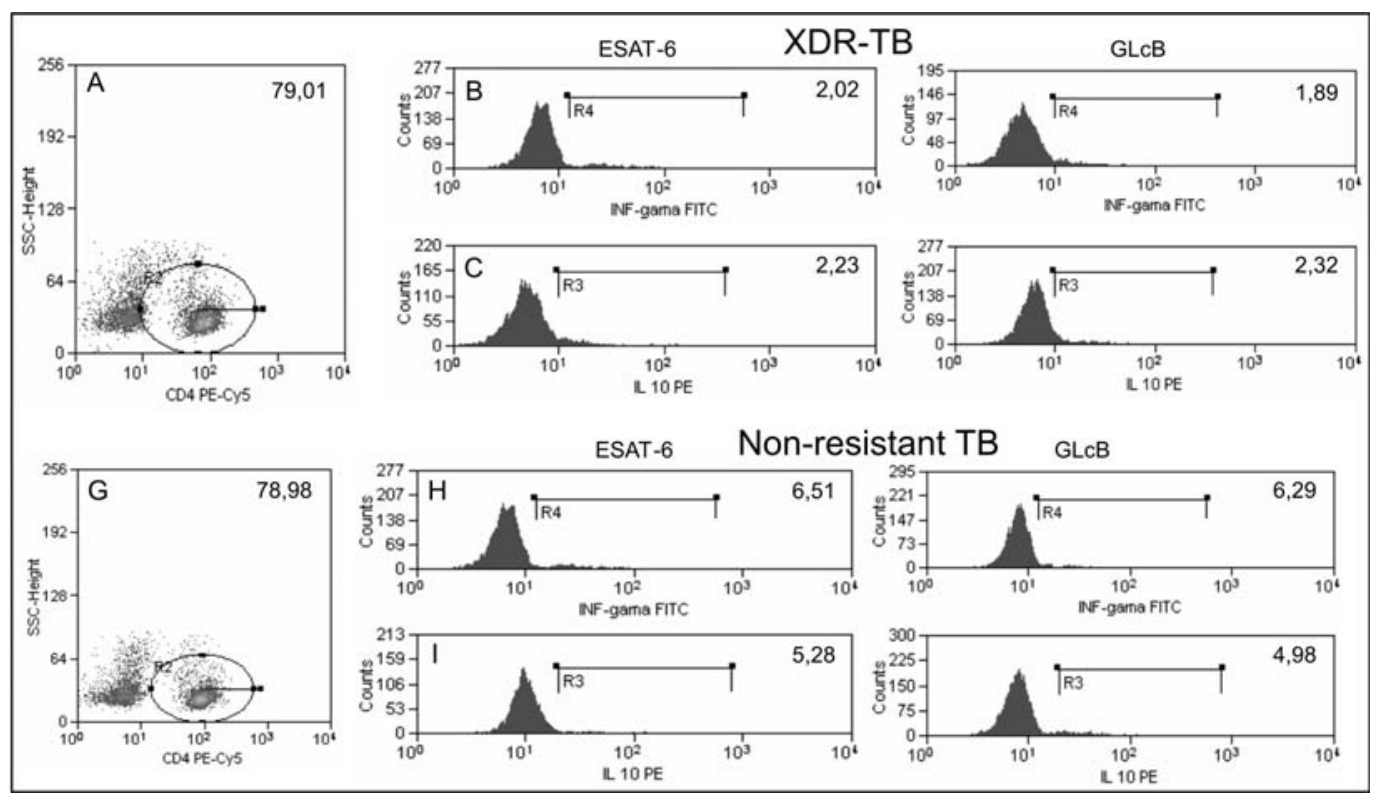

www.bjid.com.br 
The question of compulsory isolation for MDR and XDRTB cases or compulsory quarantine for suspected cases involves legal and human rights issues, and different countries will certainly approach this subject in different ways. These measures should be the last alternatives for such patients, after less restrictive alternatives fail. It is believed that compulsory isolation and quarantine alone cannot stop the spread of XDR-TB [18,23-25]; the best method to deal with $\mathrm{XDR}-\mathrm{TB}$ is prevention.

The Brazilian Constitution guarantees in its $5^{\text {th }}$ article the inviolability of one's life, freedom, equality, safety, and property, the so-called fundamental rights upon which any democracy is based. It also defines, in its $196^{\text {th }}$ article, health as the state's duty and a popular right, which "should be guaranteed through social and economic policies aiming at a decrease in disease risks and other damages and universal and egalitarian access to actions and services destined for its promotion, protection, and recuperation.” Regarding the latter article, Federal Law No. 8,080, from 9/19/1990 assigns to the national health system (SUS - Sistema Único de Saúde) the task of developing and executing actions related to the prevention and control of epidemic diseases. A number of other federal and state laws were also passed in order to regulate this obligation; they include tuberculosis in the list of diseases that must be compulsorily reported to the competent public health organs. These laws expressly allow sanitary authorities to enforce compulsory isolation in cases of patients that may represent a risk of contamination for society. In fact, the patient's wellness alone can be invoked as the reason for his/her isolation, as life, even one's own, is not disposable, according to the Brazilian legal system.

However, a systematic interpretation of these laws, taking into consideration the paramount supremacy of the constitutional right to freedom, places compulsory isolation as an extreme measure, only to be adopted when other noncoercive means fail. Furthermore, compulsory isolation must affect one's freedom only; any form of treatment that may negatively affect the patient's dignity remains absolutely illegal.

The WHO Global Task Force on XDR-TB recommends the following measures for prevention and control of XDRTB [26]. 1- Global control of TB should be strengthened immediately. 2- The algorithm and revised guidelines for diagnosis and management of patients at risk for MDR-TB and XDR-TB should be finalized and evaluated in countries without delay. 3- WHO guidelines for the programmed management of drug-resistant $\mathrm{TB}$ should be updated to address XDR-TB and TB/HIV co-management and implemented as soon as possible. Countries should consider using the Green Light Committee mechanism to facilitate access to high-quality low-priced second-line anti-TB drugs. 4- The WHO should disseminate the revised laboratory case definition for XDR-TB. A strategic, budgeted plan for laboratory strengthening should be developed at the global and national levels, with the aim of ensuring that all TB patients have access to timely, quality-assured laboratory diagnostic services; the plan should include deployment of rapid diagnostic tests. Access to second-line drug susceptibility testing should be increased. 5- Measures for infection control should be implemented rapidly in health-care settings and other high-risk areas, such as prisons, to reduce the transmission of drug-resistant TB. WHO guidelines on infection control should also be revised. 6Rapid surveys should be carried out, focusing on highrisk patients in order to determine the geographical distribution of XDR-TB. Thereafter, surveillance for XDRTB must be included within existing drug-resistance surveillance systems. 7- Advocacy, communication, and social mobilization (ACSM) should be enhanced to promote effective prevention, treatment, and control of XDR-TB at the global and national levels, especially in settings of high HIV prevalence. 8- The WHO should develop a fully budgeted plan for resource mobilization to meet the shortand long-term needs to address XDR-TB at the global, regional, and country levels. 9- The WHO should convene a committee of experts as soon as possible to review research and development issues related to XDR-TB.

This case report reminds us that implementation of highquality DOT programs is crucial to ensure proper treatment of all TB patients, in order to prevent the emergence of MDR and XDR M. tuberculosis strains.

\section{Acknowledgements}

The authors thank Dra. Eleonora Pacheco Alencastro Veiga Hsiung for providing insightful discussion about the Brazilian legal perspective on compulsory isolation and quarantine.

\section{References}

1. World Health Organization. WHO REPORT 2007: Global Tuberculosis Control: Surveillance, Planning, Financing. Geneve; 2007.

2. Maartens G., Wilkinson R. Tuberculosis. Lancet 2007;370:203043.

3. Meya D.B., McAdam K.P.W.J. The TB pandemic: an old problem seeking new solutions. J Intern Med 2004;261:309-29.

4. World Health Organization. Guidelines for the programmatic management of drug-resistant tuberculosis. Geneva: World Health Organization; 2006.

5. Center for Diseases Control and Prevention. Emergence of Mycobacterium tuberculosis with extensive resistance to secondline drugs - worldwide, 2000-2004. MMWR Morb Mortal Wkly Rep 2006;55:301-5.

6. World Health Organization. Case definition for extensively drugresistant tuberculosis. Wkly Epidemiol Rec 2006;42:408.

7. World Health Organization. Tuberculosis. XDR-TB - The facts. Available at http://www.who.int/tb/challenges/xdr/ facts_nov2007_en.pdf. Accessed January 10, 2008.

8. Gandhi N.R., Moll A., Sturm A.W., et al. Extensively drug-resistant tuberculosis as a cause of death in patients co-infected with tuberculosis and HIV in a rural area of South Africa. Lancet 2006;368:1575-80.

9. Kim H.R., Hwang S.S., Kim H.J., et al. Impact of extensive drug resistance on treatment outcomes in non-HIV-infected patients with multidrug-resistant tuberculosis. Clin Infect Dis 2007;45:1290-5. 
10. Cox H.S., Kalon S., Allamuratova S., et al. Multidrug-resistant tuberculosis treatment outcomes in Karakalpakstan, Uzbekistan: treatment complexity and XDR-TB among treatment failures. PLoS ONE 2007;2(11):e1126.

11. Raviglione M.C., Smith I.M. XDR tuberculosis - implications for global public health. N Engl J Med 2007;356(7):656-9.

12. Ebrahim G.J. Drug resistance in tuberculosis. J Trop Pediatr 2007;53:147-9.

13. Lawn S.D., Wilkinson R. Extensively drug resistant tuberculosis. A serious wake-up call for global health. BMJ 2006;333:560-1.

14. Weis S.E., Slocum P.C., Blais F.X., et al. The effect of directly observed therapy on the rates of drug resistance and relapse in tuberculosis. N Engl J Med 1994;330(17):1179-84.

15. Jasmer R.M., Seaman C.B., Gonzalez L.C., et al. Tuberculosis treatment outcomes. Directly observed therapy compared with self-administered therapy. Am J Respir Crit Care Med 2004;170:561-6.

16. Ministério da Saúde. Tuberculose multirresistente - Guia de vigilância epidemiológica. Rio de Janeiro: Ministério da Saúde; 2007.

17. Basu S., Andrews J.A., Poolman E.M., et al. Prevention of nosocomial transmission of extensively drug-resistant tuberculosis in South African district hospitals: an epidemiological modelling study. Lancet 2007;370:1500-7.
18. Goemaere E., Ford N., Berman D., McDermid C., Cohen R. XDRTB in South Africa: detention is not the priority. PLoS Medicine 2007;4(4):772-3.

19. O’brien R.J., Nunn P.P. The need for new drugs against tuberculosis. Obstacles, oppurtunities, and next steps. Am J Respir Crit Care Med 2001;162:1055-8.

20. Spigelman M., Gillespie S. Tuberculosis drug development pipeline: progress and hope. Lancet 2006;367:945-6.

21. Souza M.V.N., Vasconcelos T.R.A. Fármacos no combate á tuberculose: passado, presente e futuro. Química Nova 2005;28(4):678-82.

22. Duncan K., Barry III C.E. Prospects for new antibercular drugs. Curr Opin Microbiol 2004;7:460-5.

23. Parmet W.E. Legal power and legal rights - isolation and quarantine in the case of drug-resistant tuberculosis. N Engl J Med 2007;375(5):433-5.

24. Porco T.C., Getz W.M. Controlling extensively drug-resistant tuberculosis. Lancet 2007;370:1464-5.

25. Singh J.A., Upshur R., Padayatchi N. XDR-TB in South Africa: no time for denial or complacency. PLoS Medicine 2007;4(1):1925

26. World Health Organization. Extensively drug-resistant tuberculosis (XDR-TB): recommendations for prevention and control. Wkly Epidemiol Rec 2006;45:430-2. 